This is a self-archived version of an original article. This version may differ from the original in pagination and typographic details.

Author(s): Kari, Tuomas; Siutila, Miia; Karhulahti, Veli-Matti

Title: An Extended Study on Training and Physical Exercise in Esports

Year: 2019

Version: Accepted version (Final draft)

Copyright: @ IGI Global, 2019.

Rights: In Copyright; http://rightsstatements.org/page/InC/1.0/?language=en

Rights url:

Please cite the original version:

Kari, T., Siutila, M., \& Karhulahti, V.-M. (2019). An Extended Study on Training and Physical Exercise in Esports. In B. R. Dubbels (Ed.), Exploring the Cognitive, Social, Cultural, and Psychological Aspects of Gaming and Simulations (pp. 270-292). IGI Global. https://doi.org/10.4018/978-1-5225-7461-3.ch010 


\title{
An Extended Study on Training and Physical Exercise in Esports
}

\author{
Tuomas Kari \\ University of Jyvaskyla, Finland \\ Miia Siutila \\ University of Turku, Finland \\ Veli-Matti Karhulahti \\ University of Turku, Finland
}

\begin{abstract}
This article is an extended revision of the authors' earlier study (2016) on the training routines of professional and high-level esport players, with added focus on their physical exercise. The study is methodologically mixed with a quantitative survey sample $(n=115)$ and a qualitative interview sample $(n=7)$. Based on this data, high-level esport players train approximately 5.28 hours every day around the year and professional esport players at least the same amount. Approximately 1.08 hours of that training is physical exercise. More than half (55.6\%) of our professional and high-level esport players believe that integrating physical exercise into their training programs has a positive effect on esport performance; however, no less than $47.0 \%$ do the physical exercise chiefly to maintain their overall state of health. Accordingly, the study indicates that professional and high-level esport players are physically active as well, those of age 18 and older exercising more than three times the daily 21-minute physical activity recommendation given by the World Health Organization.
\end{abstract}

Keywords: Sport, Esport, Physicality, Training, Practice, Exercise, Empirical, Quantitative/Qualitative

\section{INTRODUCTION}

Esports have recently become a significant part of our sports cultures. A number of journalists, policymakers, and academics have thus ended up conceptualizing the cultural identity of the phenomenon: what are the relations between esports and sports, e-athletes and athletes, and esport play and physical exercise (see Taylor, 2012; Ferrari, 2013; von Hilvoorde, 2016; Freeman \& Wohn, 2017; Karhulahti, 2016; 2017; Jenny et al., 2017; Kane \& Spradley, 2017), furthermore, what are the related socio-cultural implications and what makes esport engagement rewarding to begin with? (See Lee \& Schoenstedt, 2011; Witkowski, 2012a; Harper, 2013; Martončik, 2015; Nielsen \& Karhulahti 2017; Hamari \& Sjöblom, 2017; Hallman \& Giel, 2017; Siutila \& Havaste, 2018.)

This study is not explicitly concerned with the above questions but brings them into a new light. Namely, in what follows, we explore the training routines of professional and high-level esport players with added focus on their physical exercise. This paper is an extended revision of our earlier work (Kari \& Karhulahti, 2016), enhanced by supplementary data and insights via qualitative interviews of five professional esport players. 
The solitary earlier academic contribution that we were able to find on the topic comes from Andreas Hebbel-Seeger (2012). He quotes a study from esport organization ESL (Electronic Sports League) that apparently issued an unpublished German thesis written by Lüttmann (first name unobtainable) in 2007. According to Hebbel-Seeger (2012), the study claimed that esport players are more active in sports than the average population, with no less than $95 \%$ of them exercising traditional sports as well.

Since we have not been able to acquire the referenced study (which appears to be unpublished, non-peerreviewed, and in German) we take its results with a grain of salt. For instance, it is not clear whether the study concerned professional players, high-level players, amateurs, or fans. Hence, for the present study, a priori hypotheses are not proposed. Training, physical exercise, and players' perceptions will be examined at a descriptive level without utilizing any prior theoretical framework.

\section{METHODOLOGY}

The quantitative part of the study leans on a data set that we gathered with an online survey between September 2015 and June 2016. The survey was created with the LimeSurvey 2.05+ software. We pretested it quantitatively with ten and qualitatively with four scholars from the fields of play research, videogame research, information systems research, and sports research. Based on the received feedback we made small adjustments before the launch.

The supplemental qualitative interviews were conducted in two separate occasions: June-July 2017 and April 2018. Next, we elaborate on both the quantitative and qualitative methodologies.

\section{Reaching Respondents}

The global videogame player base arguably exceeded 2B in 2016, while recent speculations talk about figures beyond 2.3B (Statista, 2018). Of those only about 9000 had ever played videogames professionally some years ago (Bräutigam, 2015), while the present number of active professional esport players, according to the website Esport Earnings, is 5950. Consequently, quantitative work on professional esport players differs significantly from that of the general player population.

We set a goal to reach a hundred professional esport players. In order to do that, we contacted 161 professional esport teams and 68 professional players directly by email and asked them to participate. As expected, only a few responded, leaving the total number of individual respondents to 31 . Notwithstanding, due to our direct contact method, we have strong reasons to believe that all the above respondents are actual professional esport players (PRO) as defined by their contracts with teams or achievements in international tournaments. Of note, for us, being a professional is thereby not defined by the player's salary but status.

Due to the low response rate we started looking for more participants via popular media in early 2016. We promoted the survey through Twitter and also posted a call on six major Reddit sub forums: Counter Strike: Global Offensive (CSGO), Dota 2 (DOTA), Hearthstone (HS), Heroes of the Storm (HOTS), League of Legends (LOL), and StarCraft 2 (SC2). This time, our goal was not to reach PRO players alone but also those who were seriously striving for a PRO career. We did, however, add requirements so that each respondent should be at the topmost tiers of the ranked player base of their esport, e.g. CSGO players were demanded to have played within the top three ranks, SC2 players were demanded to have played within the top two ranks, and LOL players we demanded to have played in Diamond 3 or higher.

Altogether the Twitter and Reddit calls reached 91 self-proclaimed high-level esport players who were competing or seriously striving to compete as professionals. We went through the data manually and 
removed seven responses that were visibly unreliable. With reference to the widely recognized psychological factors of response bias (e.g. Nederhof, 1985; Furnham, 1986; Podsakoff, MacKenzie, Lee, \& Podsakoff, 2003) and illusory superiority (e.g. Hoorens, 1995), there is a likelihood that many of these respondents have exaggerated their status. We took this into consideration by not mixing the sample with the PRO group that was reached directly, and addressing them as a distinct group of high-level players (HL) even though many of the respondents identified themselves as professionals.

In total, we thus managed to reach 115 consistent responses from PRO and HL players with various backgrounds. While the number is not high, it does derive from two relatively small target groups, which makes it unique material for analysis - quantitative studies on professional and high-level athletes being rare among sports sciences in general.

To shed light on our quantitative findings, we re-contacted some of the professional teams and individuals in order to reach them for in-depth interviews. This happened in two rounds: June-July 2017 and April 2018. During the first round, two players agreed to participate in this qualitative part of the study, followed by five more players in the second round. Hence, the total number of interviewed players is seven.

The players were all men between ages 18 and 25 . At the time of the interviews, two of them were studying in a university in the US under an esports scholarship and competing and training with success in the Top 200 of North American ranked LOL players. The other five interviewees compete in Europe, four of which being signed with teams that compete nationally and internationally in HS, Tekken, and DOTA (two players). The fifth European interviewee was a Challenger ranked LOL player in EUNE server without any active contract with a team.

\section{Survey and Interview Questions}

The survey questionnaire consisted of three main sections. The total number of questionnaire items presented to each respondent was 23, some of which were conditional. The key items are presented in Appendix A. The descriptive questions concerning training, physical exercise, and players' perceptions were all closed-ended multiple-choice questions, with the exception of the two questions regarding the hours of training to which the respondents were asked to insert a numerical value. The respondents also had the response option "cannot say" with some of the questions to avoid forced responses. We grouped the respondents into two sub-samples, PRO $(\mathrm{N}=31)$ and $\mathrm{HL}(\mathrm{N}=84)$, as explained earlier.

We used the IBM SPSS Statistics 22 software as our data analysis tool. The statistical significance and the strength of the dependencies between the responses and level of expertise (and others) were analyzed through contingency tables (crosstabs), the Pearson's $\chi 2$ tests of independence, and the Cramér's V coefficients. These methods enabled us to examine not only linear but also non-linear dependencies, which suited the explorative nature of the study well.

In some cases, the common condition for the validity of $\chi 2$ test - "No more than $20 \%$ of the expected counts are less than 5 and all individual expected counts are 1 or greater" (Yates, Moore, \& McCabe, 1999, p. 734) - was not met. Hence, as suggested by the widely used guidelines by Agresti (2002) and Cochran (1954), the results of Pearson's $\chi 2$ tests of independence were advanced by using exact tests; in this case, Monte Carlo. The Monte Carlo test was based on 10000 sampled tables and $99 \%$ confidence level. This procedure is considered reliable and independent of the dimension, allocation, distribution, and the balance of the analyzed data (Mehta \& Patel, 2012). The level of significance was set to $\mathrm{p}<0.05$.

The interview questions were designed to yield further insights on how the players train their skills in respective esport games. These questions primarily centered around the details of the players' training 
routines, their reasons for preferring some types of practice over others, and the role of physical exercise in the daily program. That said, many new questions beyond that list emerged during the sessions. Accordingly, the interviews were conducted in a semi-structured manner so that we could move flexibly from a topic to another, simultaneously allowing the interviewees to elaborate on the questions that they considered personally interesting to discuss.

\section{RESULTS}

The descriptive statistics of the quantitative sample of 115 respondents are presented in Tables $1 \mathrm{a}$ and $1 \mathrm{~b}$. The responses to the questions regarding training, physical exercise, and perceptions regarding their influence are summarized in Table 2. Table 3 summarizes the results of the Pearson's $\chi^{2}$ tests of independence and Monte Carlo exact tests, which were used to examine the statistical significance and strength of dependencies between levels of expertise (those who stated "Cannot say" were excluded).

There were no statistically significant dependencies within levels of expertise, i.e. the differences between PRO and HL regarding the responses concerning training, physical exercise, or perceptions regarding their influence were not statistically significant. Also, when classifying the respondents based on their annual esports income (those who earn less than 5000 USD and those who earn 5000 USD or more) no statistically significant dependencies between the groups arose.

Table 1a. Descriptive statistics of the whole survey sample and the sub-samples

\begin{tabular}{|c|c|c|c|c|c|c|}
\hline & \multicolumn{2}{|c|}{$\begin{array}{c}\text { Whole sample } \\
\qquad(\mathrm{N}=115)\end{array}$} & \multicolumn{2}{|c|}{$\begin{array}{c}\text { Professional } \\
(\mathbf{N}=\mathbf{3 1})\end{array}$} & \multicolumn{2}{|c|}{$\begin{array}{l}\text { High-level } \\
(\mathbf{N}=84)\end{array}$} \\
\hline & $\mathbf{N}$ & $\%$ & $\mathbf{N}$ & $\%$ & $\mathbf{N}$ & $\%$ \\
\hline \multicolumn{7}{|l|}{ Gender } \\
\hline Men & 112 & 97.4 & 29 & 93.5 & 83 & 98.8 \\
\hline Women & 3 & 2.6 & 2 & 6.5 & 1 & 1.2 \\
\hline \multicolumn{7}{|l|}{ Age } \\
\hline-19 yrs. & 50 & 43.5 & 12 & 38.7 & 38 & 45.2 \\
\hline $20-24$ yrs. & 49 & 42.6 & 16 & 51.6 & 33 & 39.3 \\
\hline $25-29$ yrs. & 12 & 10.4 & 3 & 9.7 & 9 & 10.7 \\
\hline $30-$ yrs. & 4 & 3.5 & 0 & 0.0 & 4 & 4.8 \\
\hline \multicolumn{7}{|l|}{ Yearly esports income } \\
\hline-4999 USD & 58 & 50.4 & 13 & 41.9 & 45 & 53.6 \\
\hline 5,000-14,999 USD & 16 & 13.9 & 8 & 25.8 & 8 & 9.5 \\
\hline $15,000-24,999$ USD & 8 & 7.0 & 3 & 9.7 & 5 & 6.0 \\
\hline $25,000-$ USD & 8 & 7.0 & 1 & 3.2 & 7 & 8.3 \\
\hline No answer & 25 & 21.7 & 6 & 19.4 & 19 & 22.6 \\
\hline \multicolumn{7}{|l|}{ Highest education } \\
\hline Primary education & 17 & 14.8 & 5 & 16.1 & 12 & 14.3 \\
\hline Upper secondary & 45 & 39.1 & 10 & 32.3 & 35 & 41.7 \\
\hline
\end{tabular}




\begin{tabular}{|l|l|l|l|l|l|l|}
\hline Uni of applied sciences & 26 & 22.6 & 9 & 29.0 & 17 & 20.2 \\
\hline University (BS or MS) & 21 & 18.3 & 6 & 19.4 & 15 & 17.9 \\
\hline Doctoral level & 1 & 0.9 & 0 & 0.0 & 1 & 1.2 \\
\hline Other & 5 & 4.3 & 1 & 3.2 & 4 & 4.8 \\
\hline Nationality & & & & & & \\
\hline European & 63 & 54.8 & 21 & 67.7 & 42 & 50.0 \\
\hline North-American & 35 & 30.4 & 4 & 12.9 & 31 & 36.9 \\
\hline Other & 11 & 9.6 & 4 & 12.9 & 7 & 8.3 \\
\hline No Answer & 6 & 5.2 & 2 & 6.5 & 4 & 4.8 \\
\hline
\end{tabular}

Table 1b. Descriptive statistics of the whole survey sample and the sub-samples

\begin{tabular}{|c|c|c|c|c|c|c|}
\hline & \multicolumn{2}{|c|}{$\begin{array}{l}\text { Whole sample } \\
\qquad(\mathbf{N}=115)\end{array}$} & \multicolumn{2}{|c|}{$\begin{array}{l}\text { Professionals } \\
\quad(\mathbf{N}=\mathbf{3 1})\end{array}$} & \multicolumn{2}{|c|}{$\begin{array}{l}\text { High-level } \\
(\mathbf{N}=84)\end{array}$} \\
\hline & $\mathbf{N}$ & $\%$ & $\mathbf{N}$ & $\%$ & $\mathbf{N}$ & $\%$ \\
\hline \multicolumn{7}{|l|}{ Individual vs. team } \\
\hline Individual (P vs. P) & 31 & 27.0 & 10 & 32.3 & 21 & 25.0 \\
\hline Team (team vs. team) & 78 & 67.8 & 20 & 64.5 & 58 & 69.0 \\
\hline N/A & 6 & 5.2 & 1 & 3.2 & 5 & 6.0 \\
\hline \multicolumn{7}{|l|}{ Main esport } \\
\hline Counter Strike & 51 & 44.3 & 8 & 25.8 & 43 & 51.2 \\
\hline StarCraft II & 15 & 13.0 & 7 & 22.6 & 8 & 9.5 \\
\hline DOTA 2 & 14 & 12.2 & 1 & 3.2 & 13 & 15.5 \\
\hline League of Legends & 12 & 10.4 & 7 & 22.6 & 5 & 6.0 \\
\hline Other & 23 & 20.0 & 8 & 25.8 & 15 & 17.9 \\
\hline \multicolumn{7}{|l|}{ Continent of team/self } \\
\hline Europe & 64 & 55.7 & 21 & 67.7 & 43 & 51.2 \\
\hline North America & 40 & 34.8 & 8 & 25.8 & 32 & 38.1 \\
\hline Australia & 4 & 3.5 & 1 & 3.2 & 3 & 3.6 \\
\hline Asia & 2 & 1.8 & 0 & 0.0 & 2 & 2.4 \\
\hline Other & 5 & 4.3 & 1 & 3.2 & 4 & 4.8 \\
\hline \multicolumn{7}{|l|}{$\begin{array}{l}\text { Main reason for physical } \\
\text { training }\end{array}$} \\
\hline Physical health & 54 & 47.0 & 14 & 45.2 & 40 & 47.6 \\
\hline Physical capacity & 8 & 7.0 & 1 & 3.2 & 7 & 8.3 \\
\hline Physical appearance & 20 & 17.4 & 7 & 22.6 & 13 & 15.5 \\
\hline Fun or enjoyment & 6 & 5.2 & 1 & 3.2 & 5 & 6.0 \\
\hline
\end{tabular}




\begin{tabular}{|l|l|l|l|l|l|l|}
\hline $\begin{array}{l}\text { To be more successful } \\
\text { in esports }\end{array}$ & 10 & 8.7 & 2 & 6.5 & 8 & 9.5 \\
\hline Other & 4 & 3.5 & 2 & 6.5 & 2 & 2.4 \\
\hline $\begin{array}{l}\text { Does not do any } \\
\text { physical training }\end{array}$ & 13 & 11.3 & 4 & 12.9 & 9 & 10.7 \\
\hline $\begin{array}{l}\text { Who plans physical } \\
\text { training program }\end{array}$ & & & & & & \\
\hline Myself & 81 & 70.4 & 19 & 61.3 & 62 & 73.8 \\
\hline Personal coach & 6 & 5.2 & 2 & 6.5 & 4 & 4.8 \\
\hline Team coach etc. & 5 & 4.4 & 1 & 3.2 & 4 & 4.8 \\
\hline No training plan & 21 & 18.3 & 7 & 22.6 & 14 & 16.7 \\
\hline Other & 2 & 1.7 & 2 & 6.5 & 0 & 0.0 \\
\hline
\end{tabular}

Table 2. Training, physical training, and the perceptions regarding its influence (survey)

\begin{tabular}{|c|c|c|c|c|c|c|}
\hline & \multicolumn{2}{|c|}{$\begin{array}{l}\text { Whole sample } \\
\qquad(\mathrm{N}=115)\end{array}$} & \multicolumn{2}{|c|}{$\begin{array}{l}\text { Professionals } \\
(\mathbf{N}=\mathbf{3 1})\end{array}$} & \multicolumn{2}{|c|}{$\begin{array}{l}\text { High-level } \\
(\mathbf{N}=\mathbf{8 4})\end{array}$} \\
\hline & $\mathbf{N}$ & $\%$ & $\mathbf{N}$ & $\%$ & $\mathbf{N}$ & $\%$ \\
\hline \multicolumn{7}{|l|}{ Overall training /day } \\
\hline 1-2.49 hours & 10 & 8.7 & 1 & 3.2 & 9 & 10.7 \\
\hline 2.5-4.99 hours & 42 & 36.5 & 11 & 35.5 & 31 & 36.9 \\
\hline 5-7.49 hours & 43 & 37.4 & 13 & 41.9 & 30 & 35.7 \\
\hline 7.5- hours & 20 & 17.4 & 6 & 19.4 & 14 & 16.7 \\
\hline \multicolumn{7}{|l|}{ Physical training /day } \\
\hline 0 & 18 & 15.7 & 7 & 22.6 & 11 & 13.1 \\
\hline $0<>1$ hours & 23 & 20.0 & 7 & 22.6 & 16 & 19.0 \\
\hline $1-1,5$ hours & 46 & 40.0 & 11 & 35.5 & 35 & 41.7 \\
\hline 1.51-2 hours & 23 & 20.0 & 6 & 19.4 & 17 & 20.2 \\
\hline 2- hours & 5 & 4.2 & 0 & 0.0 & 5 & 6.0 \\
\hline \multicolumn{7}{|l|}{$\begin{array}{l}\text { Influence of physical } \\
\text { training on esports }\end{array}$} \\
\hline Significantly negatively & 1 & 0.9 & 0 & 0.0 & 1 & 1.2 \\
\hline Somewhat negatively & 4 & 3.5 & 1 & 3.2 & 3 & 3.6 \\
\hline No significant effect & 21 & 18.3 & 3 & 9.7 & 18 & 21.4 \\
\hline Somewhat positively & 45 & 39.1 & 10 & 32.3 & 35 & 41.7 \\
\hline Significantly positively & 19 & 16.5 & 6 & 19.4 & 13 & 15.5 \\
\hline Cannot say & 25 & 21.7 & 11 & 35.5 & 14 & 16.7 \\
\hline
\end{tabular}




\begin{tabular}{|c|l|l|l|l|l|l|}
\hline $\begin{array}{l}\text { Physical appearance can } \\
\text { influence the performance } \\
\text { of others }\end{array}$ & & & & & & \\
\hline Yes & 34 & 29.6 & 11 & 35.5 & 23 & 27.4 \\
\hline To me & $21 / 34$ & $18.3 / 29.6$ & 7 & $22.6 / 35.5$ & 14 & $16.7 / 27.4$ \\
\hline To my opponent(s) & $27 / 34$ & $23.5 / 29.6$ & 10 & $32.3 / 35.5$ & 17 & $20.2 / 27.4$ \\
\hline No influence at all & 81 & 70.4 & 20 & 64.5 & 61 & 72.6 \\
\hline $\begin{array}{l}\text { Physical training } \\
\text { compared to teammates }\end{array}$ & & & & & & \\
\hline Significantly less & 4 & 3.5 & 2 & 6.5 & 2 & 2.5 \\
\hline Somewhat less & 7 & 6.1 & 1 & 3.2 & 6 & 7.1 \\
\hline About the same & 34 & 29.6 & 9 & 29.0 & 25 & 29.8 \\
\hline Somewhat more & 29 & 25.2 & 4 & 12.9 & 25 & 29.8 \\
\hline Significantly more & 11 & 9.6 & 4 & 12.9 & 7 & 8.3 \\
\hline N/A or cannot say & 30 & 26.1 & 11 & 35.5 & 19 & 22.6 \\
\hline
\end{tabular}

Table 3. Level of professionalism dependencies on training, physical training, and the perceptions regarding its influence (survey)

\begin{tabular}{|l|l|l|l|l|l|l|}
\hline & $\mathbf{N}$ & $\chi^{\mathbf{2}}$ & $\mathbf{d f}$ & $\mathbf{p}$ & $\mathbf{p}_{\text {(Monte Carlo) }}$ & $\mathbf{V}$ \\
\hline Overall training hours /day & 115 & 1.801 & 3 & 0.615 & 0.621 & 0.125 \\
\hline Physical training hours /day & 115 & 3.513 & 4 & 0.476 & 0.490 & 0.175 \\
\hline $\begin{array}{l}\text { Influence of physical training } \\
\text { on esports performance }\end{array}$ & 90 & 2.031 & 4 & 0.730 & 0.765 & 0.150 \\
\hline $\begin{array}{l}\text { Physical appearance can } \\
\text { influence the performance of } \\
\text { others }\end{array}$ & 115 & 0.714 & 1 & 0.398 & 0.490 (exact) & 0.079 \\
\hline
\end{tabular}

\section{Sample Analysis}

As to gender distribution, the quantitative sample is very unbalanced with only three respondents identifying as women and 112 as men. This is likely because the already-small group of professional esport players is very male-dominant (see Taylor, 2009; Maric, 2011; Chee, 2012; Zolides, 2015; Adams, 2016). We tried to fix this sample unbalance first by contacting three more institutions with striving woman esport players and later by contacting ten known successful women esport players directly. We received one reply. Therefore, the distribution did not allow any gender comparisons.

Next to the well-known male-bias, another common premise or assumption concerning esport players is age. Esport players are generally believed to be teenagers or young adults (McTee, 2014), perhaps due to the physiological changes that come along with age (see Thompson et al. 2013; 2014). Our data supports 
these assumptions, the mean age of our respondents being 20.8 years ( $\mathrm{SD}=4.4$ years) in the full quantitative sample. We should remark, however, that this age average is by no means an anomaly in sports. For instance, the average age of women swimmers in the previous Olympic finals was exactly 20.8 years (combined men and women average 21.7), whereas gymnasts are generally believed to reach their optimal level of competence at 16 or 17 with an average age of 19 in the London Olympics. Esport players are young, but so are many other athletes. We found no statistically significant differences concerning training, physical exercise, or perceptions regarding their influence between younger and older age groups.

Based on their nationalities, the respondents were distributed among three regional categories: Europe, North America, and the rest of the world. Since it is reasonable to believe that Asia covers a significant part of the professional player population (Lee, 2005; Chee \& Jin, 2008; Dongsheng Xiaohang, \& Daofeng, 2011; Szablewicz, 2011; Guorui, 2012) and only a few of the respondents identified as Asian, our data cannot be considered fully representative in this regard. As the groups of distinct nationalities were expectedly small, there is no reason to compare the practices between the represented nationalities per se. Instead, we compared the two distinguishable groups by region, North America and Europe, but found no statistically significant differences concerning training, physical exercise, or perceptions regarding their influence.

Many professional esport players do not play in their home country, but in teams and institutions around the world. These locations have their own cultural traditions and practices, which we believed to affect the players' training routines. Therefore, we additionally asked about the continent in which the respondents or their team was located. Again, the dominant continents were Europe (64) and North America (40). When comparing the responses between these two groups, the only part with a statistically significant dependency was the question whether "Physical appearance can influence the performance of others" $(\chi 2(1)=4.199, \mathrm{p}<0.04, \mathrm{~V}=0.201)$. Of Europe-based respondents $23.4 \%$ stated yes, while within North America-based players the agreement rate was $42.5 \%$. This implies that in North American esport scenes appearance - perhaps related to external play dynamics like trash-talk (see Conmy, 2008) may hold a more significant role than in those of Europe.

We also inquired about players' specific esport, but the response distribution did not allow us to draw any reasonable conclusions game-wise (44.3\% of the respondents coming from CSGO). A reasonable distinction could be made, nonetheless, between those playing team esports and those playing solo esports, the former group consisting of 78 respondents and the latter of 31 respondents. We found no statistically significant differences between these two groups concerning training, physical exercise, or perceptions regarding their influence.

Lastly, we inquired about the respondents' education level and financial income from esport sources, both questions being optional. The majority of our respondents came from primary and upper secondary schools $(53.9 \%)$, while some of them had already reached an applied sciences $(22.6 \%)$ or a university $(18.3 \%)$ degree. This makes sense with reference to their average age. Also, more than half (50.4\%) of them (including those in the PRO group respectively) declared that they earn less than 5000 USD from esport play per year, which coheres with the fact that noteworthy prize pools and salaries concern only a small number of the presently striving esport players, also among those who are signed with teams. We found no significant response variance in terms of education levels or financial compensation (cf. Parshakov \& Zavertiaeva, 2015). 


\section{Quantitative Training Analysis}

The main reason for esport players to do physical exercise is to maintain or improve overall physical health (47.0\%). This applies to both levels of expertise in our study (PRO 45.2\%, HL 47.6\%). Only 8.7\% of the respondents considered the main purpose of their physical exercise to be more successful in esport. Likewise, only $11.3 \%$ stated that they do not do any physical exercise, meaning that $88.7 \%$ of PRO and HL players do. Additionally, $81.7 \%$ claimed to have a physical exercise program. Most of the respondents (70.4\%) planned their physical exercise themselves. Only 5.2\% had a personal coach to plan the program and for $4.4 \%$ the team coach or equivalent was the planner.

The respondents did an average of 5.28 hours ( $\mathrm{SD}=2.57$ hours) of overall training per day including 1.08 daily hours ( $\mathrm{SD}=0.83$ hours) of physical exercise. Among the sub-samples, the averages were 5.90 hours and 0.89 hours for the PROs ( $\mathrm{SD}=3.07$ hours, $\mathrm{SD}=0.70$ hours) and 5.05 hours and 1.15 hours for the HLs (2.33 hours, $\mathrm{SD}=0.86$ hours).

Less than a third of the respondents (29.6\%) believed that the amount of their physical exercise was about the same as that of their teammates. A quarter of the respondents $(25.2 \%)$ believed it was somewhat more, and a tithe $(9.6 \%)$ believed it was significantly more. Lastly, another tithe $(9.6 \%)$ believed that the amount of their physical exercise was somewhat or significantly less than what their teammates did, while $26,1 \%$ could not state their belief. If these self-estimations are correct, the reached team esport players represent somewhat well the teams in which they play (with regard to questions concerning physical exercise).

As for the perceived influence of physical exercise on one's own esport performance level, most perceived it positively: either somewhat positively (39.1\%) or significantly positively (16.5\%). Only $18.3 \%$ stated not to have perceived significant effects, and $4.4 \%$ perceived the influence negatively.

We also asked the respondents whether they believed that the physical appearance of a player could influence the competitive performance of others. Less than a third (29.6\%) stated "Yes" and 70.4\% stated "No." Of all respondents, $23.5 \%$ believed that their opponent had been intimidated by their (or their teammate's) physical appearance, and $18.3 \%$ stated to have been personally intimidated by the physical appearance of their opponent.

To satisfy our special interest in the respondents' overall training hours, we ran an additional t-test over PRO and HL groups. As pointed out earlier, the t-test provided no statistically significant differences between PRO and HL groups on either overall training hours $(t=1.596, d f=113, p=0.424)$ or physical exercise hours $(\mathrm{t}=-1.518, \mathrm{df}=113, \mathrm{p}=0.498)$.

\section{Interview Analysis}

Our interviewees' training habits seem to fit relatively well with the quantitative data. Each of the interviewees were male, aged in their late teens or early 20 s, and reported doing physical exercise roughly an hour per day. There were some slight differences in the players' overall training amounts: while the first four respondents trained at least 8 daily hours, the fifth one stated to train an average of 6 hours per day, and the remaining two reported four or five-hour daily minimums. As a caveat, each player noted that they typically took one day off every week. Again, the average amount of daily training in these statements lines well with our quantitative results.

Next to playing the game, the interviewees stated that a significant amount of their training time is spent on other activities related to the game such as watching their own replays and other professional play. 
They also stressed that discussing and theorizing the games with the team and other peers was a daily routine; moreover, they also sought game-related information on a regular basis. The players had difficulties in estimating how much time they spent on these non-play activities, yet the standard guess (excluding the HS player) was half of the training time being spent on playing and the rest on everything else.

The most deviant answers regarding play and training came from the one HS player who reported only two hours of actual daily play. He considered Hearthstone to be very different compared to other esport games: "mechanics are not really important in Hearthstone like in other games." It is possible that being successful in HS requires less play time and more theorycrafting as well as strategic planning. When these activities are taken into account, his daily averages reach the same numbers (6) as the other players.

In response to a question whether the 12-14 hours per day that professional esports athletes are often reported to train (e.g. DiChristopher, 2014; Jacobs, 2015; Stanton, 2015) were truthful or exaggerated, the interviewees unanimously considered them truthful. One of the DOTA players, for instance, pointed out that his team had recently been advised to increase their amount of daily training significantly, as others simply "train more than you and do a better job at it."

In coherence with the quantitative finding that players exercise physically largely in order to maintain their overall health, all of the interviewees considered physical exercise to be helpful in esports through generally better health and more active lifestyle. Good physical health was said to increase concentration, mood, and energy levels, thus helping to focus through daily training and tournaments.

Despite the players themselves believing in the benefits of physical exercise, their coaches and teams put relatively little emphasis on it: for those with a team contract, the minimum amount of physical exercise that the players were encouraged to do weekly was only one or two hours. In other words, the players did significantly more physical exercise contra what their coaches and teams entailed. Our interviewees did not have a physical exercise plan; instead, they engaged in various different activities. While one reported trying to "take a 20-30-minute jog twice a day," another had weights and a power wheel at home that he would use "four to five times a week about 20 minutes at a time" in addition to going for daily walks. A third one played soccer (in a team) a few times a week, and a fourth reported "working out about four times a week" next to the mandatory physical training with the esport team once per week (involving "varying activities for an hour or two"). The interviewee with the least physically intensive exercise routine reported that he most often just went for about 30-minute walks daily and sometimes did other activities like going to the gym.

We also asked about exercise as a form of preventing typical esport injuries such as those related to wrists or neck and shoulder pain. Only one of the respondents reported actively trying to remember to stretch his wrists and "move around" between games, while the others only expressed having knowledge of these things being helpful. They were more concerned about passive preventative measures like having proper gaming chairs and setup in general. The little rehabilitative or preventive exercise that was done was occasional and mostly related to already feeling some pain or stiffness ("if my neck or shoulders hurt I'll get up and move" and "stretching is good if there are problems with wrists").

There is an interesting discrepancy in the players' attitudes towards physical exercise and the amount of exercise they (are required to) do. For instance, one player described physical exercise to be possibly the most overlooked aspect of training in esports ("real physical exercise is underrated and not understood well enough how significant it is"), but at the same time admitted his own physical exercise to be somewhat random. While players clearly believe in the potential benefits of physical exercise in terms of esport performance, the physical exercise they factually do seems to be largely unstructured and unsystematic. 


\section{CONCLUSION}

We conclude by summarizing three points that we consider most significant.

First, the study implies that the overall time that professional and high-level esport players spend on training might be heavily exaggerated by the popular press. While journalists and other media contributors frequently present these numbers around 12 or 14 daily hours, the average of our survey respondents was "only" 5.28 hours. There are multiple potential explanations for this. Initially, it is possible that players who take their esport seriously are engaged with the activity all around the clock, but only part of that time gets spent on actual play. In other words, the players might well spend 12-14 daily hours on related activities such as team meetings, video analysis, strategic discussions, sponsored events, interviews, and so on; however, what they count as training is solely the time they play or physically exercise. This was partially supported by the interviews, as the players explicitly stressed that their daily amounts of training (as stated) were only hard minimums and that they probably trained a lot more in reality (not excluding days that were supposed to be dedicated to rest). Another possible explanation is that the high numbers presented in various media concern merely the very top of esport players, a large part of which reside in Asia and were thus not represented manifestly in our samples.

Second, the study implies that professional and high-level esport players are relatively active also when it comes to physical exercise. On average, such players appear to exercise physically 1.08 hours every day. This is more than the World Health Organization's (2018) physical activity recommendation for both children of 5-17 years (60 minutes daily) and adults of 18-64 years (21 minutes daily). Keeping in mind that the average age of our respondents is 20.8 , it appears that adult esport players do physical exercise more than three times the recommended amount. When it then comes to the balance between the physical activity recommendations of children and the training requirements of high-level play, we thus submit that, from the present viewpoint, the age restrictions set by many esport tournaments and leagues (usually at the minimum of 17) are accurate. We also entertain the possibility that the non-trivial amounts of physical exercise in top-ranked players' training regimes might have positive effects on the physical activity behaviors of amateurs and new players who see them as idols or role models (e.g. Dix, Phau \& Pougnet, 2010).

Third, the study implies that the reasons behind professional and high-level esport players' relatively high physical exercise amounts are not so much due to their desire to improve competitive performance, but rather in their awareness concerning the benefits of healthy lifestyles. Almost half of the respondents $(47.0 \%)$ considered the upkeep of their overall health as the main reason for their daily physical exercise, whilst more than half $(55.6 \%)$ believed it to have a positive side effect on their competitive careers as well. As we disagree with scholars who decline all parallels "in the physical efforts of competitive athletes and e-athletes" (Wimmer, 2012, p. 533) and agree with those who consider the physical involvement of esport to be "identifiable not just in quick hands or self-control [but also in] managing and engaging with multiple bodily senses and actions" (Witkowski, 2012b, p. 362), it seems that the greatest potential of esport as a physical cultural practice lies in its tendency to inform those involved about the benefits of bodily routines.

While regular physical exercise may or may not have direct positive effects on esport performance, those who play esport on higher levels seem to have grasped the likely advantages that regular physical exercise generates for their overall health mentally and physically (see Warburton, Nicol, \& Bredin, 2006). However, despite understanding the importance and possible positive effects of regular exercise, there seems to be a lack of knowledge concerning what kinds of exercise, how much of it, and how often it should be engaged in. Furthermore, the value of exercise as a means to prevent injuries may not have reached an optimal level yet. As for the ongoing and future debates concerning the "physicality" of esport and its suitability for children in particular, we would be less concerned about the time that aspiring 
young players spend training inside, and more concerned about the building of social structures that help those players recognize the advantages of training outside too as early as possible.

\section{LIMITATIONS AND FUTURE RESEARCH}

Our study has evident limitations that should be noted. The first one relates to the quantitative operationalization of the surveyed concepts (e.g. "physicality") in a relatively simplistic manner, measured by single item measures. Future research could use more rigorous operationalization by measuring concepts with multiple questions that would enable evaluating their reliability and validity. Likewise, a limitation derives also from the fact that the measures were based on the respondents' subjective perceptions. Future studies could benefit from actually following esport players and teams, thus using more objective measures to track their general training and the role of physical exercise in it.

Our study did not include any exergame players (see Kari, 2014; Kari \& Makkonen, 2014). This deficiency can also be seen as a strength, as the physical activity patterns of exergame players presumably differ significantly from those studied above. It would probably be best to study them as a distinct player group. That said, the respondent numbers and variance could have been greater in general. With greater numbers and variance, potential gender differences would be a substantial subject of study. Likewise, a parallel study on Asian esport players in particular should provide an interesting point of comparison.

We also entertain the possibility that dedicated videogame play (and other forms of active play) might be related to physical activity more generally. For instance, it is possible that individuals who establish longterm ludic relationships with any kind of gaming are inclined to do regular physical exercise in the same way as professional and high-level esport players do. The hypothesis is intriguing, and a highly promising subject for follow-up studies.

Regardless of these limitations, our results should stand as a decent basis for future research. We specifically look forward to work with other methodologies and more advanced analysis tools. It would also be interesting to find out how much the training routines of the herein studied players differ from those of amateur and casual esport players. Comparative studies concerning the training of esports, motorsports, shooting sports, chess, and other activity groups with alike competitive requirements would likely yield fascinating results.

\section{ACKNOWLEDGEMENTS}

The study was partially supported by the Foundation for Economic Education. The authors would like to thank all esport players who participated in the study. Thanks go also to the people who provided public critical feedback after the publication of the first version of this study.

\section{REFERENCES}

Adams, J. L. (2016). Female Fighters: Perceptions of Femininity in the Super Smash Bros. Community. Press Start, 3(1), 99-114.

Agresti, A. (2002). Categorical data analysis, New York, NY: Wiley.

Bräutigam, T. (2015, September 15). Esports Statistics: The Growth of Our Industry in Five Charts. The Esports Observer. Retrieved from: http://esportsobserver.com/esports-statisticsthe-growth-of-ourindustry-in-five-charts/ 
Chee, F. (2012). Online Games as a Medium of Cultural Communication: An Ethnographic Study of Socio-Technical Transformation. Doctoral Dissertation. Fraser University.

Cochran, W. G. (1954). Some methods for strengthening the common $\chi 2$ tests. Biometrics, 10(4), 417451.

Conmy, O. (2008) Trash Talk in a Competitive Setting: Impact on Self-Efficacy, Affect, and Performance. Doctoral Dissertation. Florida State University.

DiChristopher, T. (2014, February 3). Pro gamers story: Get big, burn out, retire young. CNBC. Retrieved from http://www.cnbc.com/2014/02/01/pro-gamers-story-get-big-burn-out-retireyoung.html.

Dix, S., Phau, I., \& Pougnet, S. (2010). "Bend it like Beckham": the influence of sports celebrities on young adult consumers. Young Consumers, 11(1), 36-46.

Dongsheng, Y., Xiaohang, Y., \& Daofeng, K. (2011). The Present Situation and Development Trend of E-sports Games in China. In 2011 International Conference on Future Computer Science and Education (ICFCSE) (pp. 384-386). IEEE.

Ferrari, S. (2013). eSport and the Human Body: Foundations for a Popular Aesthetics. In Proceedings of DiGRA 2013: DeFragging Game Studies, August 26-29. Atlanta, GA: DiGRA.

Freeman, G. \& Wohn, D. (2017). eSports as an Emerging Research Context at CHI: Diverse Perspectives on Definitions. In Proceedings of the 2017 CHI Conference Extended Abstracts on Human Factors in Computing Systems (pp. 1601-1608). ACM.

Furnham, A. (1986). Response bias, social desirability and dissimulation. Personality and Individual Differences, 7(3), 385-400.

Guorui, Z. (2012). Bibliometric Analysis on E-Sports in China. In Advances in Computer Science and Engineering (pp. 111-118). Springer.

Hallman, K. \& Giel, T. (2017). eSports-Competitive Sports or Recreational Activity? Sport Management Review. First Online.

Hamari, J., \& Sjöblom, M. (2017). What is eSports and why do people watch it? Internet Research, 27(2), (advance online publication).

Harper, T. (2013). The Culture of Digital Fighting Games: Performance and Practice, New York, NY: Routledge.

Hebbel-Seeger, A. (2012). The relationship between real sports and digital adaptation in e-sport gaming. International Journal of Sports Marketing and Sponsorship, 13(2), 43-54.

Hoorens, V. (1995). Self-favoring biases, self-presentation, and the self-other asymmetry in social comparison. Journal of Personality, 63(4), 793-817.

Jacobs, H. (2015, May 11). Here's the insane training schedule of a 20-something professional gamer. Business Insider. Retrieved from: http://www.businessinsider.com/pro-gamersexplain-the-insanetraining-regimen-they-use-to-stay-on-top-2015-5?r=US\&IR=T\&IR=T. 
Jenny, S., Manning, R., Keiper, M. \& Olrich, T. (2017). Virtual(ly) Athletes: Where eSports Fit within the Definition of "Sport". Quest, 69(1), pp. 1-18.

Lee, A. (2005). E-Sports as a Growing Industry. (Research Report). Samsung Economic Research Institute.

Lee, D., \& Schoenstedt, L. J. (2011). Comparison of eSports and traditional sports consumption motives. The ICHPER-SD Journal of Research in Health, Physical Education, Recreation, Sport \& Dance, 6(2), 39.

Kane, D. \& Spradley, B. (2017). Recognizing Esports as a Sport. Sport Journal. First Online.

Karhulahti, V. (2016). Prank, Troll, Gross and Gore: Performance Issues in Esport Livestreaming. In Proceedings of the 1st International Joint Conference of DiGRA and FDG. Digital Games Research Association and Society for the Advancement of the Science of Digital Games.

Karhulahti, V. (2017). Reconsidering Esport: Economics and Executive Ownership. Physical Culture and Sport: Studies and Research, 74 (1), 43-53.

Kari, T. (2014). Can Exergaming Promote Physical Fitness and Physical Activity?: A Systematic Review of Systematic Reviews. International Journal of Gaming and Computer-Mediated Simulations, 6(4), 5977.

Kari, T. \& Karhulahti, V. (2016) "Do E-Athletes Move? A Study on Training and Physical Exercise in Elite E-Sports.” International Journal of Gaming and Computer-Mediated Simulations, 8.

Kari, T., \& Makkonen, M. (2014). Explaining the Usage Intentions of Exergames. In Proceedings of the 35th International Conference on Information Systems (ICIS) 2014. AIS.

Maric, J. (2011). Electronic Sport: How Pro-gaming Negotiates Territorial Belonging and Gender. Platform: Journal of Media and Communication, ECREA Special Issue, 3(2), 6-23.

Martončik, M. (2015). E-Sports: Playing Just for Fun or Playing to Satisfy Life Goals? Computers in Human Behavior, 48, 208-211.

McTee, M. (2014). E-Sports: More Than Just a Fad. Oklahoma Journal of Law \& Technology, 10, 1-27.

Mehta, C. R., \& Patel, N. R. (2012). IBM SPSS Exact Tests. Cambridge, MA: IBM Corporation.

Nederhof, A. J. (1985). Methods of coping with social desirability bias: A review. European Journal of Social Psychology, 15(3), 263-280.

Nielsen, R. \& Karhulahti, V. (2017). The Problematic Coexistence of Internet Gaming Disorder and Esports. In Proceedings of the 12th International Conference on the Foundations of Digital Games. ACM.

Parshakov, P., \& Zavertiaeva, M. A. (2015). Success in eSports: Does Country Matter?, SSRN Scholarly Paper 2662343. Retrieved from: http://papers.ssrn.com/abstract=2662343 (accessed 2 March 2016). 
Podsakoff, P. M., MacKenzie, S. B., Lee, J. Y., \& Podsakoff, N. P. (2003). Common method biases in behavioral research: a critical review of the literature and recommended remedies. Journal of Applied Psychology, 88(5), 879-903.

Siutila, M., Havaste, E. (2018) "A pure meritocracy blind to identity": Exploring the Online Responses to All-Female Teams in Reddit, in Proceedings of the 2018 DiGRA International Conference

Stanton, R. (2015, June 22). The secret to eSports athletes' success? Lots - and lots - of practice. ESPN. Retrieved from: http://www.espn.com/espn/story/_id/13053116/esports-athletes-puthours-training-reachpinnacle.

Statista. (2018). The Statistics Portal. Statista.com.

Szablewicz, M. (2011). From Addicts to Athletes: Participation in the Discursive Construction of Digital Games in Urban China. In Selected Papers of Internet Research 12.0 (pp. 1-21). Association of Internet Researchers.

Taylor, N. (2009). Cheerleaders, Booth Babes, Halo Hoes: Pro-gaming, Gender and Jobs for the Boys. Digital Creativity, 20(4), 239-252.

Taylor, T. L. (2012). Raising the Stakes: E-Sports and the Professionalization of Computer Gaming. Cambridge, MA: The MIT Press.

Thompson J. J., Blair M. R., Chen L., \& Henrey A. J. (2013). Video Game Telemetry as a Critical Tool in the Study of Complex Skill Learning. PLoS ONE, 8(9), e75129.

Thompson J. J., Blair M. R., \& Henrey A. J. (2014). Over the Hill at 24: Persistent Age-Related Cognitive-Motor Decline in Reaction Times in an Ecologically Valid Video Game Task Begins in Early Adulthood. PLoS ONE, 9(4), e94215.

von Hilvoorde, I. (2016). Sport and Play in a Digital World. Sport, Ethics and Philosophy.

Warburton, D., Nicol, C., \& Bredin, S. (2006). Health benefits of physical activity: the evidence. Canadian Medical Association Journal, 174, 801-809.

Wimmer, J. (2012). Digital Game Culture(s) as Prototype(s) of Mediatization and Commercialization of Society. In J. Fromme \& A. Unger (Eds.), Computer Games and New Media Cultures: A Handbook of Digital Game Studies (pp. 525-540). Heidelberg: Springer.

Witkowski, E. (2012a). Inside the Huddle: The Phenomenology and Sociology of Team Play in Networked Computer Games (Doctoral Dissertation). IT University of Copenhagen.

Witkowski, E. (2012b). On the Digital playing Field: How we "Do Sport" With Networked Computer Games. Games and Culture, 7(5), 349-374.

World Health Organization (WHO). (2018). Global recommendations on physical activity for health. (Report). WHO.

Yates, D., Moore, D., \& McCabe, G. (1999). The Practice of Statistics. New York, NY: W.H. Freeman. 
Zolides, A. (2015). Lipstick Bullets: Labour and Gender in Professional Gamer Self-Branding. Persona Studies, 1(2), 42-53.

\section{KEY TERMS AND DEFINITIONS}

Esports: cultural practices of exercise and contest on commercial play products that are governed by executive owners

High-level esport player: seriously striving to become a professional player and performing similar activities as professional players, but having not yet reached international competence or team contracts

Physical exercise: activity requiring physical effort, carried out to sustain or improve health and fitness

Practice: performing certain activities in order to become better at them, sometimes synonymous with training

Professional esport player: individual competing in sanctioned international leagues or tournaments, or playing in an officially registered team with a contract

Training: the process of learning or becoming better at specific skill or skills, sometimes synonymous with practice 


\section{APPENDIX: KEY SURVEY QUESTIONS REGARDING TRAINING AND ESPORTS ${ }^{1}$}

1. How many hours of training do you do daily? (Hours per day on average)

Total amount of all training related to being a better or more successful player.

You can also report uneven hours by using a dot. For example: 1 hours 30 minutes $=1.5$ (hours) and 30 minutes $=0.5$ (hours).

2. How many hours of PHYSICAL training do you do daily? (Hours per day on average) For example: running, cycling, strength, gym-training, yoga, etc.

You can also report uneven hours by using a dot. For example: 1 hours 30 minutes $=1.5$ (hours) and 30 minutes $=0.5$ (hours).

3. Who plans your physical training program?

$\circ \quad$ I do it myself o My personal coach

- Team's head coach o Team's physical coach/physiotherapist

0 My team buys this as a service from outside

- I have no physical training plan. I just do whatever and whenever I feel like it

O Other: [specify your choice in the accompanying text field]

4. What is your MAIN reason for doing physical training?

○ To maintain or improve my overall physical health

- To maintain or improve my physical capacity

- To lose weight, gain muscles, or tone my body (physical appearance)

- For fun or enjoyment of exercising

- To be more successful in esports

- I don't do any physical training

- Cannot say

O Other: [specify your choice in the accompanying text field]

5. How do you perceive that doing PHYSICAL training has affected your performance level in esports?

○ Significantly negatively

- Somewhat negatively

- No significant effect

- Somewhat positively

- Significantly positively

- Cannot say

6. Compared to your teammates, do you believe you are doing more or less physical training than they do?

- Significantly less

- Somewhat less

- About the same amount

- Somewhat more

- Significantly more

- Cannot say 
7. Do you believe that the physical appearance of a player can influence the competitive performance of others?

Please choose all that apply:

$\square \quad$ Yes, I believe my opponent has been intimidated by my (or my teammate's) physical appearance

$\square$ Yes, I have been intimidated by the physical appearance of my opponent

$\square$ No, I don't believe that there is any influence

Choose all 'Yes' -options that apply OR 'No' -option.

\section{ENDNOTES}

${ }^{1}$ Detailed descriptions of all the other questions are available from the authors by request 